\title{
Spiral computed tomography for preoperative staging of potentially resectable carcinoma of the pancreatic head
}

\author{
S. S. K. S. Phoa, J. W. A. J. Reeders, E. A. J. Rauws*, L. de Wit $\dagger$, D. J. Gouma $\dagger$ and J. S. Laméris \\ Departments of Radiology, *Gastro-enterology and $†$ Surgery, Academic Medical Centre, Meibergdreef 9, 1105 AZ, Amsterdam, The Netherlands \\ Correspondence to: Dr S. S. K. S. Phoa
}

\begin{abstract}
Background: Pancreatic cancer is often locally invasive. Preoperative staging attempts to identify patients suitable for resection, in order to minimize unnecessary operations. The aim of this study was to assess the improved imaging provided by spiral computed tomography (CT) in the preoperative staging of potentially resectable pancreatic head carcinoma.

Methods: In 56 consecutive patients with pancreatic head carcinoma spiral CT findings were correlated prospectively with operative and histopathological findings. Criteria for irresectability at CT were infiltration of the peripancreatic fat and vascular ingrowth grade $D$, on a scale from $A$ to $F$.

Results: At operation 27 (48 per cent) of 56 tumours were irresectable. Small metastases were found in seven patients (12 per cent). Ingrowth (adherence) to the portal or mesenteric vein was present in 19 patients (34 per cent). The sensitivity and specificity of CT for irresectability were 78 and 76 per cent respectively. Resection rates with a vascular margin free of tumour were 100 per cent for grade $A, 63$ per cent for grade B, 44 per cent for grade $C, 15$ per cent for grade $D$ and 0 per cent for grade $E$, with a predictive value for ingrowth of 88 per cent for grades $D$ or higher. The resectability rate was 11 per cent (one of nine) when infiltration of the anterior peripancreatic fat was present and 67 per cent when infiltration was absent $(P<0 \cdot 01)$.

Conclusion: Spiral CT with thin slices seems to improve detection of distant metastases and vascular ingrowth in patients with pancreatic head carcinoma.
\end{abstract}

Paper accepted 15 February 1999

British Journal of Surgery 1999, 86, 789-794

\section{Introduction}

Patients with a pancreatic head carcinoma have a poor prognosis and the only chance of cure is surgical resection. Preoperative staging focuses on detection of non-resectable disease to prevent unnecessary laparotomy ${ }^{1-3}$. Before the introduction of spiral computed tomography (CT), dynamic CT was regarded the best technique in staging pancreatic head carcinoma with an accuracy of $90-100$ per cent for predicting irresectable tumours ${ }^{2,3}$. However, the predictive value for resectability was as low as 28 per cent ${ }^{4}$. The predictive value for resectability was reported to be slightly better ( 56 per cent) for spiral CT, in a series using sections $8 \mathrm{~mm}$ thick $^{5}$. Small metastases and vascular ingrowth in portal or mesenteric veins accounted for approximately 40 per cent each in causing a false-negative spiral CT scan ${ }^{4}$. Detection of liver lesions and visualization of anatomical details of the pancreas and peripancreatic vessels can be improved using thinner sections in spiral $\mathrm{CT}^{6,7}$.
The aim of this study was to evaluate the use of spiral CT with thin sections $(5 \mathrm{~mm})$ for staging of patients with potentially resectable malignancy in the pancreatic head region. The CT findings were correlated with findings at operation and histopathological examination in 56 patients with pancreatic head carcinoma, who underwent resection with curative intent.

\section{Patients and methods}

Between June 1995 and December 1996, 113 consecutive patients suspected to have pancreatic head carcinoma underwent both spiral CT and duplex sonography as preoperative assessment.

In 14 patients benign disease was diagnosed: chronic focal pancreatitis in seven, obstructing bile duct stones in three and no pancreatic abnormality in four. In eight patients percutaneous biopsy proved metastases to the liver or distant lymph nodes. In 16 patients the pancreatic mass was considered irresectable, owing to the local extent of tumour, 
vascular occlusion or a perivascular mass, with narrowing of the vessel and an abnormal Doppler shift. Ten patients presumed to have resectable tumours were unfit for surgery $(n=1)$, refused operation $(n=1)$ or were treated at other institutions $(n=8)$.

The remaining 65 patients underwent diagnostic laparoscopy with laparoscopicsonography and subsequentplanned laparotomy for attempted resection. In two patients no final diagnosis was obtained (in one extensive pancreatitis prohibited resection and one patient died from cholangitis before operation). In two patients operation was delayed for more than 2 months after CT. Five patients underwent resection but no carcinoma was found (one carcinoid tumour and four cases of chronic focal pancreatitis).

This study included the remaining 56 patients, with proven pancreatic head carcinoma, in whom CT could be correlated with surgical findings. There were $34 \mathrm{men}$ and 22 women, with a mean age of 60 (range 40-76) years.

\section{Spiral computed tomography}

Spiral CT was performed on a Siemens Somatom Plus scanner. Unenhanced contiguous 10-mm slices of liver and pancreas were followed by contrast-enhanced spiral CT of the pancreas ( $5 \mathrm{~mm}$ slice thickness, 24 rotations, pitch 1 ). Some $130 \mathrm{ml}$ Megluminejoxithalamathe $300 \mathrm{mg} / \mathrm{ml}$ (Guerbet, Paris, France) was infused at $2 \mathrm{ml} / \mathrm{s}$; the scan delay was $55 \mathrm{~s}$. A second spiral CT with $5-\mathrm{mm}$ slice thickness was made through the liver.

\section{Computed tomography staging}

CT examinations were scored prospectively by a radiologist, who was blinded to all clinical and diagnostic information. Obstruction of the bile or pancreatic duct and the presence of a mass in the pancreatic head was noted.

Tumours were graded according to Loyer et . $^{8}$ : grade A, fat plane visible between tumour and vessel; grade $B$, normal pancreatic tissue between tumour and vessel; grade C, tumour adjacent to vessel with a convex contour towards vessel; grade $\mathrm{D}$, tumour adjacent to vessel with a concave contour towards vessel; grade E, circumferential involvement of vessel; and grade F, vascular occlusion. Tumours were scored as irresectable if infiltration of peripancreatic fat planes was present or when involvement of the portal vein or superior mesenteric vein was graded as $\mathrm{D}$ or higher. Lesions were also scored as irresectable when arterial encasement was present: complete circumferential involvement (cuff sign), narrowing or occlusion of the artery. All other lesions were scored as resectable, including liver lesions that could not be punctured percutaneously.
At laparoscopy with laparoscopic sonography and at surgical exploration tumour irresectability owing to metastases or local extent was always confirmed with biopsies. CT findings were correlated with findings at laparoscopy, surgical exploration and histopathological findings. Evidence of tumour adherence to the portal or mesenteric vein was regarded as tumour ingrowth; decisions about resectability and vein resection were made on the basis of macroscopic appearances.

Statistical analysis was by $\chi^{2}$ test with one degree of freedom.

\section{Results}

\section{Computed tomography diagnosis}

In 54 of the 56 malignant lesions CT demonstrated a mass in the pancreatic head. In two patients CT demonstrated only dilatation of both pancreatic and common bile ducts. Fortynine (91 per cent) of the 54 lesions detected were qualified by CT as malignant (hypodense lesion, clearly demarcated from normal pancreas, with ductal obstruction). In five cases distinction from pancreatitis was not possible. The mean diameter of the tumours visible at CT was 2.8 (range $1-4.5) \mathrm{cm}$. On pathological examination the mean size of resectable lesions was $3 \mathrm{~cm}$.

\section{Surgical findings}

Twenty-seven (48 per cent) of 56 carcinomas were irresectable; liver metastases were found in six patients (11 per cent) and peritoneal metastases in one. One patient also had distant malignant lymph nodes. In 21 patients without liver metastases, local irresectability was proven with biopsies at trial dissection (19 had venous involvement).

Twenty-nine (52 per cent) of the 56 carcinomas were resectable, and a resection was performed. Three patients underwent a sleeve resection of the vein, owing to tumour ingrowth detected at a late phase of the resection (after transection of the pancreas).

\section{Computed tomography staging}

CT data were correlated with overall resectability at surgical exploration, including patients with metastases. In 28 patients $C T$ scored the tumour as resectable, correctly in 22 (79 per cent); six were proven irresectable owing to vascular ingrowth. CT graded 28 tumours as irresectable, correctly in 21 patients. In seven a resection could be performed (three with tumour-positive vascular resection margins). All six patients with liver metastases were in the 
group scored as irresectable. Sensitivity, specificity, and positive and negative predictive values of CT for irresectability at surgery were 78 per cent ( 21 of 27$), 76$ per cent ( 22 of 29), 75 per cent (21 of 28$)$ and 79 per cent (22 of 28$)$ respectively. Excluding the patients with metastases (who did not undergo trial dissection of the vein), CT findings are correlated with local surgical resectability in Table 1. The sensitivity and positive predictive value for local irresectability were slightly lower than those for overall resectability (71 and 68 per cent respectively).

A radical resection (tumour-negative resection margins at pathology) was obtained in 20 of 29 resected tumours, including one case of sleeve resection (Fig. 1). In Table 2 resectability at $\mathrm{CT}$ is correlated with radicality of the resection, excluding the patients with metastases. The positive predictive value of $\mathrm{CT}$ for a non-radical resection was 82 per cent, with a sensitivity of 60 per cent. If patients with metastases were included, the overall positive predictive value of CT for a non-radical resection was 86 per cent, with a sensitivity of 67 per cent.

Table 1 Resectability at computed tomography correlated with local surgical resectability

\begin{tabular}{lccc} 
& \multicolumn{2}{l}{ Surgery } & \\
\cline { 2 - 3 } & Resectable & Irresectable & Total \\
\hline CT & & & \\
Resectable & 22 & 6 & 28 \\
Irresectable & 7 & 15 & 22 \\
Total & 29 & 21 & 50
\end{tabular}

The sensitivity, specificity, and positive and negative predictive values of computed tomography (CT) for local irresectability at operation were 71 per cent ( 15 of 21$), 76$ per cent ( 22 of 29$), 68$ per cent ( 15 of 22$)$ and 79 per cent (22 of 28 ) respectively

Table 2 Resectability at computed tomography correlated with radicality on pathological examination

\begin{tabular}{llll} 
& $\begin{array}{l}\text { Radical } \\
\text { resection }\end{array}$ & $\begin{array}{l}\text { Non-radical } \\
\text { resection }\end{array}$ & Total \\
\hline CT & & & \\
Resectable & 16 & 12 & 28 \\
Irresectable & 4 & 18 & 22 \\
Total & 20 & 30 & 50
\end{tabular}

The sensitivity, specificity, and positive and negative predictive values of computed tomography (CT) for a non-radical resection at pathological examination were 60 per cent (18 of 30), 80 per cent (16 of 20), 82 per cent (18 of 22) and 57 per cent (16 of 28) respectively

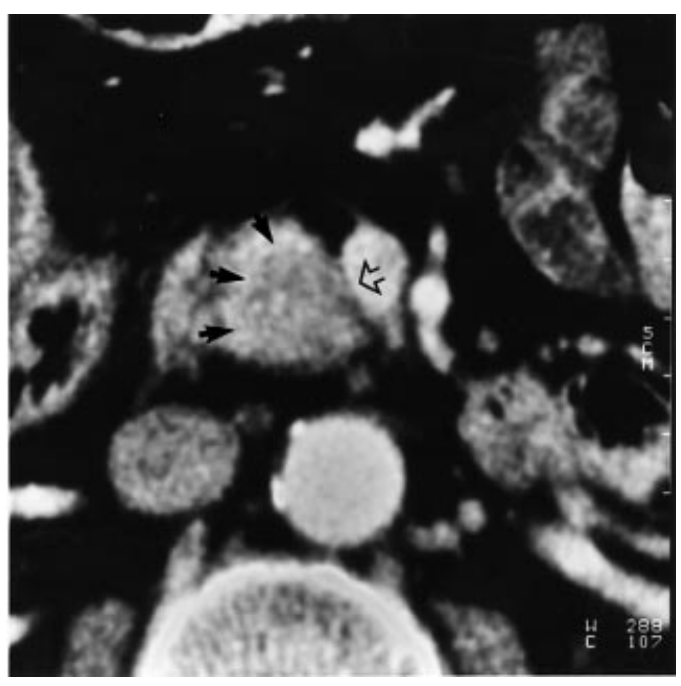

Fig. 1 Small pancreatic head carcinoma (black arrows). Computed tomography grade $\mathrm{C}$ contact with superior mesenteric vein with flattening of the vein (open arrow). The lesion was radically resected; sleeve resection was necessary

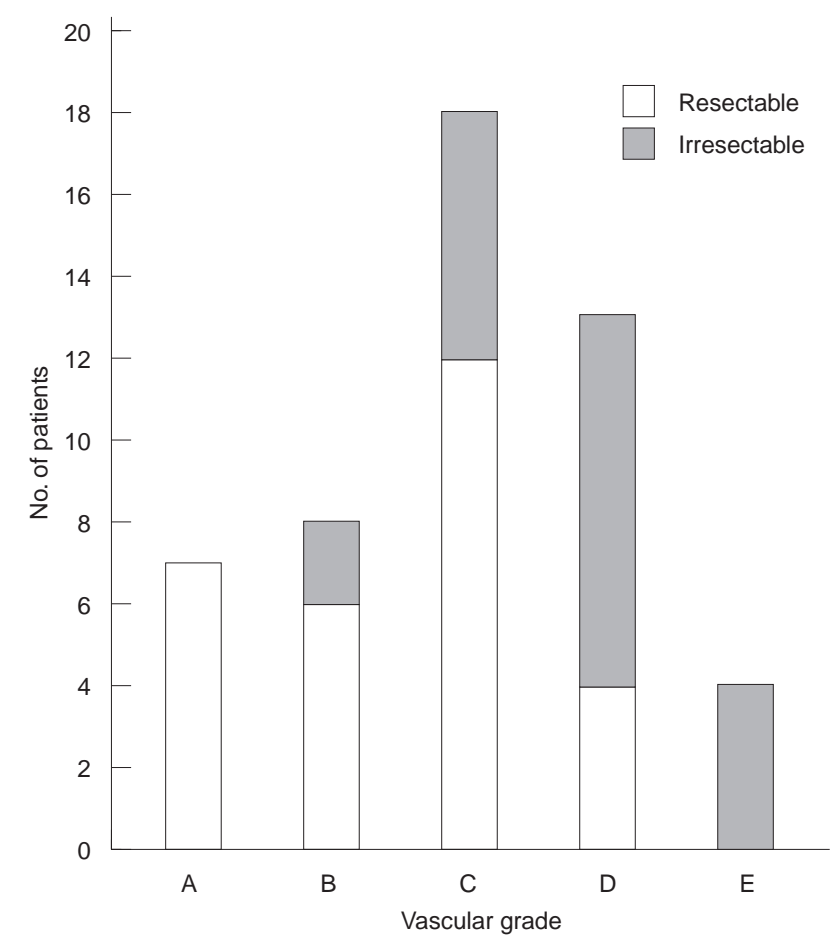

Fig. 2 Computed tomography (CT) grade of venous involvement correlated with local surgical resectability. The sensitivity, specificity, and positive and negative predictive values of CT for local irresectability at surgery (CT grade D and E) were 62, 86, 76 and 76 per cent respectively $(n=50)$ 


\section{Vascular grading by computed tomography}

In 19 patients local irresectability at operation was due to venous ingrowth. Arterial ingrowth was found in eight patients, but never without coexisting venous ingrowth. Excluding patients with metastases and using the highest CT grade for portal vein and superior mesenteric vein as a single parameter, surgical resection rates for grades A-E were 100, 75, 67, 31 and 0 per cent respectively (Fig. 2). The resection rate was 76 per cent ( 25 of 33$)$ in grades A-C and 24 per cent (four of 17$)$ at grades $\mathrm{D}$ and $\mathrm{E}(P<0.001)$.

Venous ingrowth on histopathological examination could be assessed in 50 patients, after resection or if biopsies of the vascular plane were taken during trial dissection. A sleeve resection was considered as ingrowth, regardless of resection margins. Venous ingrowth was thus found in 15 of 17 tumours with CT grades D or E, yielding a predictive value of 88 per cent (Figs 3 and 4). Resection rates with a vascular margin free of tumour were 100 per cent for grade A, 63 per cent for grade B, 44 per cent for grade C, 15 per cent for grade $\mathrm{D}$ and 0 per cent for grade $\mathrm{E}$. Infiltration of peripancreatic fat planes was one of the CT parameters for

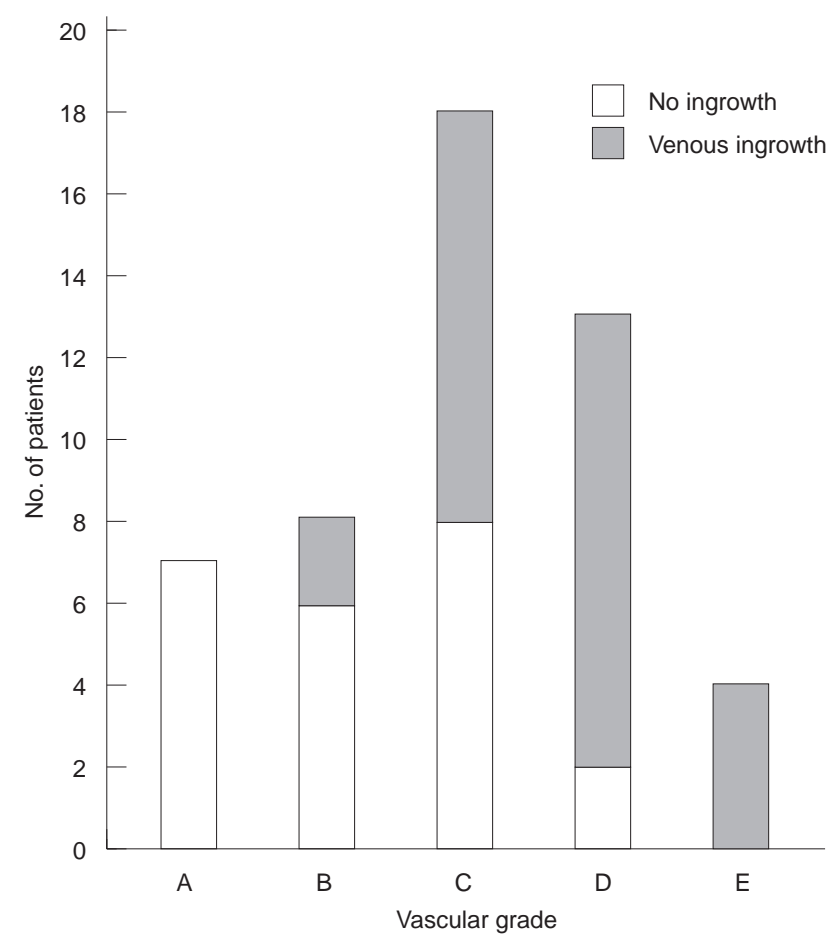

Fig. 3 Computed tomography (CT) grade of venous ingrowth correlated with vascular ingrowth at pathological examination. The sensitivity, specificity, and positive and negative predictive values of CT (grades D and higher) for venous ingrowth at pathological examination were 56,91, 88 and 64 per cent respectively local irresectability. Correlation with local surgical resectability was examined for patients without metastases. Infiltration of the anterior peripancreatic fat plane was seen in nine patients and a resection could be performed in only one. If anterior fat plane infiltration was absent the resectability rate was 67 per cent $(P<0.01)$. Posterior fat infiltration was present in 16 patients and seven of these underwent resection (resection margins were free of tumour in five).

There were seven patients with indeterminate liver lesions at CT which could not be biopsied percutaneously. The lesions were smaller than $15 \mathrm{~mm}$ in two, less than $10 \mathrm{~mm}$ in two and less than $5 \mathrm{~mm}$ in three. Two lesions were proven malignant at laparoscopic sonography and four were shown to be benign; one lesion had negative biopsies at laparoscopic sonography, but proved malignant after a short follow-up.

\section{Discussion}

CT has been regarded as the most accurate diagnostic modality in preoperative staging of pancreatic head carcinoma. The sensitivity and specificity for irresectability have been reported to be as high as 100 per cent $(\text { Table } 3)^{5,9-12}$. The findings from the present study seem comparable to those of McCarthy et al. ${ }^{9}$, but are less accurate than those in studies using a $3-\mathrm{mm}$ helical technique $^{10}$. Results should be interpreted cautiously as variation in resectability rates could indicate differences

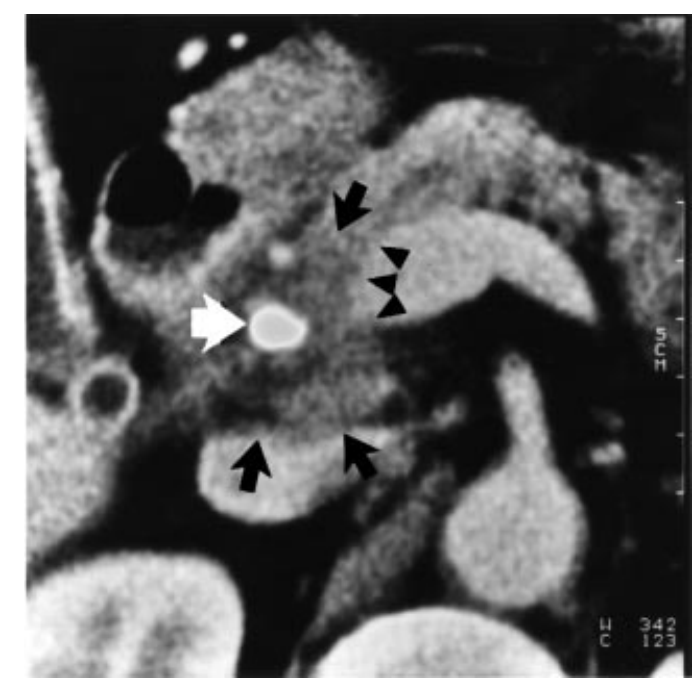

Fig. 4 Pancreatic head carcinoma (black arrows). Computed tomography grade $\mathrm{D}$ vascular ingrowth with concave contour of the tumour towards the venous confluence (arrowheads). Stent in common bile duct (white arrow). At operation the lesion was irresectable owing to vascular ingrowth 
Table 3 Sensitivity and specificity of computed tomography for irresectability correlated with surgery

\begin{tabular}{|c|c|c|c|c|c|c|c|}
\hline Reference & Year & $\begin{array}{l}\text { Sensitivity } \\
(\%)\end{array}$ & $\begin{array}{l}\text { Specificity } \\
(\%)\end{array}$ & $\begin{array}{l}\text { No. of } \\
\text { patients }\end{array}$ & $\begin{array}{l}\text { Resection } \\
\text { rate (\%) }\end{array}$ & Lesion & Technique \\
\hline McCarthy et al..$^{9^{*}}$ & 1998 & 72 & 80 & 67 & 48 & Pancreatic carcinoma & \\
\hline Diehl et al..$^{10}$ & 1998 & 91 & 90 & 76 & 28 & Pancreatic carcinoma & Dual slice $3 \mathrm{~mm}$ \\
\hline Bluemke et al..$^{5}$ & 1995 & 53 & 100 & 64 & 34 & Pancreatic carcinoma & Spiral $8 \mathrm{~mm}$ \\
\hline Megibow et al. ${ }^{4}$ & 1995 & 77 & 50 & 143 & 18 & Pancreatic neoplasms & Dynamic $5 \mathrm{~mm}$ \\
\hline Warshaw et al. ${ }^{11}$ & 1990 & 56 & 87 & 55 & 29 & Pancreatic head carcinoma & Dynamic \\
\hline Freeny et al. ${ }^{12}$ & 1988 & 95 & 100 & 51 & 3 & Pancreatic carcinoma & Dynamic $10 \mathrm{~mm}$ \\
\hline Present series & 1999 & 78 & 76 & 56 & 52 & Pancreatic head carcinoma & Spiral $5 \mathrm{~mm}$ \\
\hline
\end{tabular}

${ }^{*}$ Retrospective study

in patient selection or surgical strategy. In the present series with 5 -mm slice thickness, small metastases were undetected or unproven after CT in 12 per cent of patients. As only patients with biopsy-proven metastases were excluded from further investigation, this seems an improvement in preoperative detection of metastases compared with that in series using dynamic $\mathrm{CT}^{11}$ or helical CT with 8-mm slice thickness. Metastases were found at operation in 40 per cent of patients considered resectable at $\mathrm{CT}$ in one series ${ }^{5}$. This improved detection of metastases may partly result from improved fine-needle biopsies often performed under CT or ultrasonographic guidance, but may also be due to patient selection.

Laparoscopy and laparoscopic sonography has been advocated in preoperative staging ${ }^{13-15}$. This technique has recently been shown to demonstrate metastases that were undetected before operation in 35 per cent of patients with pancreatic head carcinoma ${ }^{16}$. Laparoscopy with sonography and diagnostic puncture may be useful in patients with indeterminate liver lesions at CT, which cannot be punctured percutaneously. In this study such patients were regarded as potentially resectable and received further investigation by laparoscopy. CT has been reported to detect a large number of small benign lesions ${ }^{17}$; the present data agree with this finding (four of seven indeterminate lesions proved to be benign).

Vascular encasement is the major cause of local irresectability and is found in approximately 50 per cent of patients thought resectable after $\mathrm{CT}^{5}$. Data are hard to compare as different CT criteria have been used. When correlating findings with surgical resectability, results are also dependent on varying attitudes towards venous resection ${ }^{18}$. Complete encirclement of the vessels and total occlusion are considered 100 per cent specific for irresectability, but these criteria are not found in many patients. In a study that used thin- section helical CT, vascular involvement was found in 88 per cent of patients with more than $180^{\circ}$ of vessel encirclement ${ }^{19}$. In the present series this criterion represented 100 per cent surgical irresectability, although it was present only in grade $\mathrm{E}$ tumours (with complete circumferential involvement of the vessel). In grade D tumours (concave contour of tumour towards vessel) the resectability rate was also low (31 per cent) and all lesions had less than $180^{\circ}$ of venous involvement. If radicality is also taken into account, a resection with tumour-free vascular margins could be obtained in only 15 per cent of grade D tumours. It is questionable whether this criterion should be used to exclude patients from resection.

The sensitivity of 56 per cent for vascular ingrowth seems low when correlating CT with pathological examination. This may partly be due to exclusion of patients who have evident vascular encasement at preoperative ultrasonography and CT, and exclusion of patients with encasement on CT without histological confirmation because metastases were found at surgery. In nearly one-third of patients who underwent resection, vascular ingrowth was found at pathological examination. Microscopic ingrowth therefore seems hard to predict at surgery as well. The predictive value of CT for vascular ingrowth at surgery was 76 per cent. A recent study, using 3-mm spiral CT, found a predictive value for ingrowth of 70 per cent for axial CT compared with surgery. In the same study threedimensional rendering of vessels showed a predictive value for ingrowth of 90 per cent $^{20}$.

In conclusion, spiral CT with thin slices seems to improve detection of liver metastases and vascular ingrowth in patients with carcinoma of the pancreatic head. Further studies should be performed to find definitive criteria that can exclude patients from laparotomy, because of a high predictive value for ingrowth. 


\section{References}

1 Kelly DM, Benjamin IS. Pancreatic carcinoma. Ann Oncol 1995; 6: 19-28.

2 Reznek RH, Stephens DH. The staging of pancreatic adenocarcinoma. Clin Radiol 1993; 47: 373-81.

3 Nghiem HV, Freeny PC. Radiologic staging of pancreatic adenocarcinoma. Radiol Clin North Am 1994; 32: 71-9.

4 Megibow AJ, Zhou XH, Rotterdam H, Francis IR, Zerhouni EA, Balfe DM et al. Pancreatic adenocarcinoma: CT versus MR imaging in the evaluation of resectability - report of the Radiology Diagnostic Oncology Group. Radiology 1995; 195: 327-32.

5 Bluemke DA, Cameron JL, Hruban RH, Pitt HA, Siegelman $\mathrm{SS}$, Soyer $\mathrm{P}$ et al. Potentially resectable pancreatic adenocarcinoma: spiral CT assessment with surgical and pathologic correlation. Radiology 1995; 197: 381-5.

6 Zeman RK, Silverman PM, Cooper C, Weltman DI, Ascher SM, Patt RH. Helical (spiral) computed tomography. Implications for imaging of the abdomen. Gastroenterol Clin North Am 1995; 24: 183-99.

7 Winter TC, Nghiem HV, Schmiedl UP, Freeny PC. CT angiography of the visceral vessels. Semin Ultrasound CT MR 1996; 17: 339-51.

8 Loyer EM, David CL, Dubrow RA, Evans DB, Charnsangavej C. Vascular involvement in pancreatic adenocarcinoma: reassessment by thin-section CT. Abdom Imaging 1996; 21 : 202-6.

9 McCarthy MJ, Evans J, Sagar G, Neoptolemos JP. Prediction of resectability of pancreatic malignancy by computed tomography. Br 7 Surg 1998; 85: 320-5.

10 Diehl SJ, Lehmann KJ, Sadick M, Lachmann R, Georgi M. Pancreatic cancer: value of dual-phase helical CT in assessing resectability. Radiology 1998; 206: 373-8.

11 Warshaw AL, Gu Z-Y, Wittenberg J, Waltman AC.
Preoperative staging and assessment of resectability of pancreatic cancer. Arch Surg 1990; 125: 230-3.

12 Freeny PC, Marks WM, Ryan JA, Traverso LW. Pancreatic ductal adenocarcinoma: diagnosis and staging with dynamic CT. Radiology 1988; 166: 125-33.

13 Andren-Sandberg A, Lindberg CG, Lundstedt C, Ihse I. Computed tomography and laparoscopy in the assessment of the patient with pancreatic cancer. 7 Am Coll Surg 1998; 186: $35-40$.

14 van Delden OM, de Wit LT, Bemelman WA, Reeders JW, Gouma DJ. Laparoscopic ultrasonography for abdominal tumor staging: technical aspects and imaging findings. Abdom Imaging 1997; 22: 125-31.

15 van Dijkum EJ, de Wit LT, van Delden OM, Rauws EA, van Lanschot JJ, Obertop H et al. The efficacy of laparoscopic staging in patients with upper gastrointestinal tumors. Cancer 1997; 79: 1315-19.

16 John TG, Greig JD, Carter DC, Garden OJ. Carcinoma of the pancreatic head and periampullary region. Tumor staging with laparoscopy and laparoscopic ultrasonography. Ann Surg 1995; 221: 156-64.

17 Jones EC, Chezmar JL, Nelson RC, Bernardino ME. The frequency and significance of small (less than or equal to $15 \mathrm{~mm}$ ) hepatic lesions detected by CT. A7R Am $\mathcal{F}$ Roentgenol 1992; 158: 535-9.

18 Harrison LE, Brennan MF. Portal vein resection for pancreatic adenocarcinoma. Surg Oncol Clin N Am 1998; 7: 165-81.

19 Lu DS, Reber HA, Krasny RM, Kadell BM, Sayre J. Local staging of pancreatic cancer: criteria for unresectability of major vessels as revealed by pancreatic-phase, thin-section helical CT. AfR Am 7 Roentgenol 1997; 168: 1439-43.

20 Raptopoulos V, Prassopoulos P, Chuttani R, McNicholas MM, McKee JD, Kressel HY. Multiplanar CT pancreatography and distal cholangiography with minimum intensity projections. Radiology 1998; 207: 317-24. 